\title{
Práctica docente en contextos multiculturales: Lecciones para la formación en competencias docentes interculturales
}

\author{
Practice Teaching in Multicultural Contexts: \\ Lessons to Training in Intercultural Teaching Skills
}

\author{
Cristina Escalante Rivera' \\ Ministerio de Educación Pública \\ San José, Costa Rica \\ crisescriv@hotmail.com \\ David Fernández Obando² \\ Ministerio de Educación Pública \\ San José, Costa Rica \\ davidestebanf@gmail.com \\ Marcelo Gaete Astica ${ }^{3}$ \\ Ministerio de Educación Pública \\ San José, Costa Rica \\ mgaetea43@gmail.com
}

Recibido 21 de noviembre de 2013 • Corregido 13 de marzo de 2014 • Aceptado 2 de abril de 2014

1 Psicóloga. Licenciada en Psicología. Departamento de Estudios e Investigación Educativa (DEIE) Ministerio de Educación Pública. Participación en diversas investigaciones del DEIE no publicadas. Colaboración para la ejecución de diferentes estudios e investigaciones con enfoques cualitativos y cuantitativos en el área de mercadeo. Cruz Roja Costarricense en la Unidad de Soporte Psicológico 2008-2010, experiencia en apoyo psicológico, psicoterapia y elaboración de proyectos. Costa Rica.

2 Orientación. Licenciado en Educación con énfasis en Orientación. Investigador Departamento de Estudios e Investigación Educativa. Asesor de la Viceministra Académica de Educación en el período 2006-2010. Ejecución de distintos estudios en su actual departamento, no publicados. Costa Rica.

3 Antropología. Máster en Antropología. Investigador Departamento de Estudios e Investigación Educativa (DEIE) Ministerio de Educación Pública. Participación en diversas investigaciones del DEIE no publicadas. Costa Rica. 
doi: http://dx.doi.org/10.15359/ree.18-2.4

URL: http://www.una.ac.cr/educare

CORREO: educare@una.cr

Resumen. El presente artículo proviene de una investigación titulada Ejercicios docentes en contextos multiculturales: Lecciones para la formación en competencias docentes interculturales, realizada durante el 2011-2012, por el Departamento de Estudios e Investigación Educativa del Ministerio de Educación Pública (Escalante, Fernández y Gaete, 2012), con la finalidad de explorar la diversidad cultural en las aulas e instituciones educativas en Costa Rica. Este fenómeno multicultural ha obligado a prestar especial atención a la oferta educativa que se brinda, principalmente, en primaria. Además, ha generado la discusión con respecto a los vacíos conceptuales y pedagógicos en el personal y en sus habilidades y destrezas en el proceso de enseñanza de estas poblaciones de origen distinto. De igual manera se impone una reflexión de los currículos educativos, los que resultan mayoritariamente nacionales y básicos. El estudio se realizó en 12 instituciones de primaria de diferente direcciones regionales, que tienen una alta diversidad cultural entre el alumnado. Por medio de técnicas cualitativas de investigación se explora las opiniones de directores, docentes y estudiantes al respecto. La conclusión más importante a la que se ha llegado es la ausencia de una pedagogía intercultural en las aulas nacionales y la necesidad de preparar al cuerpo docente en este sentido.

Palabras claves. Formación inicial docente, competencias docentes, educación intercultural.

Abstract. This paper presents the results of a research project entitled Teaching Exercises in Multicultural Contexts: Lessons to Training in Intercultural Teaching Skills, which was conducted during 20112012 by the Department of Teaching Research and Studies from the Costa Rican Ministry of Public Education (Escalante, Fernández and Gaete, 2012), in order to explore cultural diversity in classrooms and educational institutions in Costa Rica. This multicultural phenomenon has forced authorities to pay special attention to the educational services provided, particularly in elementary. In addition, it has sparked a discussion regarding the teachers' conceptual and pedagogical void and a gap in their teaching skills to deal with student populations of different origins. Similarly, it leads to a reflection about the basic national educational curriculum. The research was conducted in 12 elementary schools from different educational districts, which have a high cultural diversity among students. Using qualitative research techniques, the opinions of principals, teachers and students regarding this topic are explored. The most important conclusion reached in this study is the absence of an intercultural pedagogy in the country's classrooms and the need to prepare teachers in this respect.

Keywords. Initial teaching training, teaching skills, intercultural education.

En la actualidad, los cambios políticos y económicos han generado migraciones desde distintas regiones en el mundo y han provocado un sistema educativo culturalmente diverso, en el cual Costa Rica no es la excepción. Se evidencia, por tanto, la necesidad de actuar de acuerdo con esta realidad y brindar a estas poblaciones una adecuada oferta educativa.

De esta forma, el Departamento de Estudios e Investigación Educativa (DEIE) del Ministerio de Educación Pública (MEP) propone una investigación dirigida a examinar los ejercicios docentes en contextos multiculturales, la cual forma parte del proyecto de la CECCSICA denominado Mejoramiento de las condiciones académicas y técnicas en que se llevan a cabo en los procesos de formación inicial de docentes para atender la educación inicial y la educación primaria o básica en la región de Centroamérica y la República Dominicana, llevado a cabo desde octubre de 2011 a octubre de 2012. 
Es esta dirección, el objetivo de este estudio fue comprender las formas de atención de la diversidad en 12 instituciones educativas del país, así como también identificar los vacíos que se deben llenar en la formación inicial docente y que son pieza clave en la adquisición de las competencias necesarias para la atención de la multiculturalidad en el aula. Con ello, se pretende una reflexión para fomentar cambios curriculares que enriquezcan los planes de estudio de las universidades públicas y para valorar las experiencias positivas de aquellas instituciones que han trabajado sobre el tema.

\section{El enfoque intercultural}

El enfoque de interculturalidad supone una estrategia racional para la integración social y cultural tanto de las minorías étnicas, como de las de nacionales migrantes y nacionales sociales, en el entendido de que trasciende el modelo de aculturación unidimensional, en el cual se busca la integración total del otro cultural, hacia un modelo bidimensional, en el que se busca la integración de la persona distinta, pero respetando su cultura.

Ello ha implicado, necesariamente, que las distintas sociedades deban poner en práctica procesos mediante los cuales se integra o asimila a las personas inmigrantes. Al menos dos macro procesos de aculturación se ponen en práctica societalmente: uno que se llama unidimensional y el otro denominado bidimensional. El primero implica la pérdida de la identidad original, para adquirir la nueva, la de contacto; en el otro, se busca que haya identificación con la nueva cultura, aunque sin pérdida de la propia (Núñez, 2009).

El modelo bidimensional -"de ida y vuelta" entre el individuo y la cultura dominantecontiene 4 enfoques de aculturación, tanto de estrategias que pone en práctica el inmigrante como de las que lleva a cabo la sociedad receptora.

En lo referente a las personas inmigrantes:

a) Integrativo. Comparte la identidad con la sociedad receptora, pero mantiene su identidad propia, bidireccionalidad. Es la más utilizada.

b) Separativa. La preocupación por el mantenimiento de la propia cultura le hace rechazar el contexto de la cultura dominante o país receptor.

c) Asimilación. No quiere mantener su cultura y prefiere adoptar la de la sociedad receptora.

d) Marginalista. No se identifica ni con la cultura propia ni con la de la sociedad receptora. Problemas de estrés, autoestima y adaptación (Núñez, 2009).

Por otra parte, la sociedad receptora pone en práctica también distintas estrategias de aculturación, siempre en la lógica supuesta de la bidireccionalidad, las cuales son: 
doi: http://dx.doi.org/10.15359/ree.18-2.4

URL: http://www.una.ac.cr/educare

CORREO: educare@una.cr

a) Multiculturalismo: Mutua acomodación del grupo dominante y la dominación.

b) Segregación: La cultura de la sociedad receptora no busca relacionarse con la cultura del migrante, del otro, lo segrega. Modelo visto en las sociedades con "Apartheid!".

c) El modelo del "Melting pot" o "Pressure Cook"s: Propicia la asimilación forzada del inmigrante, con independencia de la cultura del migrante. Se observa en los barrios segregados de algunas sociedades.

d) Y el modelo de exclusión: Hay desacuerdo en que los inmigrantes mantengan su cultura propia (Núñez, 2009).

Naturalmente, ninguna de las estrategias se presenta en forma pura en la realidad, sino en una interacción sinérgica. El Modelo de Berry, citado por Núñez (2009), puede ayudar a entender estos dinamismos multiculturales en cuanto la intersección de modelos individuales y societales. Berry (citado por Nuñez, 2009) plantea las relaciones entre el contacto cultural y las necesidades de mantenimiento de la cultura, cruzamiento que define distintos modelos de integración cultural: Multiculturales, asimilacionistas (Melting pot), segregacionistas y excluyentes.

De este modo, entre los modelos integracionistas y segregacionistas puede darse una gama importante de modelos de aculturación social: unas sociedades estarán más cerca de uno que de otro, serán más o menos tolerantes o plurales e integradas, y permitirán mayor o menor interculturalidad. De estos, el horizonte más afirmativo consiste en alcanzar las mayores cotas de interculturalidad posibles.

Es decir, el desarrollo de las competencias interculturales entre el personal docente debe inscribirse en el campo en que se cruzan las estrategias integrativas de los migrantes y otras culturas, con las estrategias sociales del multiculturalismo, lo cual supone, por otro lado, un alto componente de interculturalidad que, para el caso de este estudio, supone la estructuración de procesos de capacitación docente en las competencias interculturales en el aula.

\section{De los modelos de aculturación social a los modelos de educación multicultural}

El actual contexto multicultural requiere de una educación adecuada que se ubique en el contexto de lo universal/particular, de lo homogéneo/heterogéneo y de lo local/global; fenómenos polares que afectan las relaciones entre los individuos, las poblaciones, etnias y grupos culturales. Este contexto ha generado intolerancia (Merino y Muñoz, 1998).

4 Nombre dado al tipo de régimen político en que vivió Sudáfrica, caracterizado por la segregación y discriminación de la población negra.

5 Expresiones que se utilizan para referirse a la idea de crisol de etnias en Estados Unidos, en donde la mezcla es la característica principal, y del hecho de que este crisol de etnias convive bajo la presión de la intolerancia racial. 
Por otra, los procesos migratorios cuestionan el modelo de sociedad tradicional homogéneo y obliga a dar respuesta cultural a este hecho, respuesta que la encontramos en el proceso de socialización, el cual, contemporáneamente, se puede visualizar como una función de la educación (Merino y muñoz, 1998).

No obstante, este proceso de socialización cultural, o mejor dicho en una terminología más apropiada de aculturación, que es una función educacional, se expresa por medio de diversos modelos educativos de atención de la diversidad cultural que identifican los antropólogos García, Pulido y Montes (1997), y detallamos seguidamente:

a) Educar para igualar. La asimilación cultural es el centro funcional de este modelo. Hay que asimilar para mejorar el éxito de niños diferentes. La escuela opera como el medio que hace transitar a los estudiantes hacia su igualación cultural.

b) Entendimiento cultural. La diferencia hay que conocerla, hay una valoración de las diferencias culturales. La escuela se orienta al enriquecimiento cultural.

c) Pluralismo cultural. Preservar y extender el pluralismo. La escuela debe preservar el pluralismo cultural, pero las minorías no toleran la asimilación.

d) La educación bicultural. Hacer estudiantes competentes en dos culturas: la receptora y la del inmigrante. El modelo se centra en la preservación de la lengua propia.

e) La educación como transformación. Educación multicultural y reconstrucción social. Desarrollo de la conciencia cultural del estudiantado y de su entorno familiar para lograr el empoderamiento sociocultural del estudiantado y su familia respecto de la realidad.

f) Educación antirracista. La educación multicultural antirracista es ideológica y radical y está fundada en el análisis de clases sociales.

En todos estos modelos de multiculturalidad hay una suposición de fondo y es que las diferencias de la niñez en la escuela son básicamente culturales, suposiciones que en el fondo vuelven la cultura, la identidad cultural como una falencia. Tal suposición y corolario impide ver el efecto que por encima de la cultura tienen, en la educabilidad, los procesos de desigualdad social, y con ello la idea de que no todas las culturas son válidas para el desarrollo social occidental, por lo que la asimilación es la respuesta para la equiparación educativa (García et al., 1997).

Un modelo propuesto desde la antropología cultural, más actual, reconoce la cultura como un atributo humano de diferenciación y de organización colectiva de la diferencia, encarnada en la cultura del grupo (García et al., 1997), procesos que son también resultado de los fenómenos de la dinámica y estructura social.

Esta visión agrega que la cultura es una covalidación significativa de sí misma, de la cultura propia y de la de otros. Acepta que la multiculturalidad constituye un hecho, dado que 
doi: http://dx.doi.org/10.15359/ree.18-2.4

URL: http://www.una.ac.cr/educare

CORREO: educare@una.cr

obliga a que todos los seres humanos desarrollen competencias en diversas culturas, y que cada ser humano tiene acceso a varias culturas, o microculturas, es decir, "a más de un conjunto de conocimientos y patrones de percepción, pensamiento y acción" (García et al., 1997, p. $240)$, se impone modelar el proceso de aculturación de modo sinérgico mediante procesos de educación intercultural.

\section{Pedagogía intercultural y competencias}

El enfoque teórico que orienta esta investigación se condensa en la discusión que actualmente se suscita en torno al concepto de pedagogía intercultural, entendiendo lo pedagógico como el proceso de mediación cognoscitivo del estudiantado, desde el reconocimiento de la diversidad y lo intercultural como diálogo que se debe propiciar en el centro educativo $y$, en particular, en el aula, entre los grupos sociales y culturales presentes en esa realidad, hasta una habilidad dialogal, la cual requiere de competencias especificas interculturales (Rehaag, 2010).

\section{El concepto de competencia}

El concepto de competencia se entiende como "... autoridad, capacidad profesional, competición, cualificación, incumbencia e incluso como suficiencia ... respecto al grado de preparación, de saber hacer; los conocimientos y pericia para desarrollar las tareas y funciones..." (Lévy-Leboyer, 1996, citado por Aneas, 2005, p. 1-2) asignadas. También como cualidades y condiciones profesionales que, referidas a las situaciones sociales culturalmente diversas 0 interculturales, siguiendo a Aneas, implicarían una persona que es "... hábil, eficiente o apta para desarrollar sus tareas y funciones en contextos profesionales multiculturales (...)" (Aneas, 2005, p. 2). Tal persona es poseedora de tales capacidades.

La capacidad o competencia intercultural"comporta toda una serie de requisitos, actitudes, destrezas y conocimientos que son los que permiten, a dicha persona, dar una respuesta adecuada a los requerimientos de tipo profesional, interpersonal y afectivo que emergen de los citados contextos multiculturales" (Aneas, 2005, p. 2); debido a que tales capacidades no son innatas, hay que instalarlas (Rehaag, 2010).

En dos dimensiones, las competencias interculturales deben ser instaladas en las personas: en cuanto la cualificación profesional y psicoafectiva. Estas se relacionan con lo que Aneas (2005) denomina bases psicosociales de las relaciones interculturales. La última tiene que ver con una observación realizada respecto de las emociones que sienten las personas cuando se enfrentan a un entorno multicultural y se encuentran con otros diferentes. Aneas (2005, p. 3, citando a Stephan, Stephan y Gudykunst, 1999a, p. 613) rescata la siguiente apreciación respecto al estado de ánimo de una persona que se enfrenta a un entorno cultural distinto: 
Cuando personas que proceden de diferentes grupos interactúan, experimentan de un modo u otro cierta preocupación. Preocupación que puede deberse a la posibilidad de no ser suficientemente capaces para desenvolverse, preocupación de salir perjudicado del encuentro, preocupación por poder ser víctima de malos entendidos, afrentas, etc. La ansiedad generada por todas estas posibilidades puede crear en sí misma dificultades y afectos que dificulten dicha relación.

Tales sensaciones y ambivalencias se resuelven en parte cuando las personas son interculturalmente competentes y disponen:

... del conocimiento acerca de su propia cultura y de otras diferentes. El manejo de un segundo idioma ayuda mucho para la comunicación e interacción con personas de otras culturas $y$, por lo mismo, es indispensable para la competencia intercultural ... Se espera una posición sin prejuicios frente a otras culturas, así como la disposición a aprender de los "otros" (policentrismo). (Rehaag, 2010, p. 78)

Lo anterior puede ser llevado a cabo a través de la ejecución de ciertas subcompetencias, las cuales se concentran en distintas áreas, que al ser puestas en práctica, permiten a las personas acercarse de manera más efectiva a las otras culturas con las que pudiese estar teniendo contacto. Estas subcompetencias son, según lo expuesto por Rehaag (2010):

Competencia lingüística. Conocimiento suficiente del idioma del país o del lugar.

Competencia del área. Conocimientos acerca de la geografía y cultura del país o lugar, y también un conocimiento metódico de la organización e investigación.

Competencia social. Empatía y entendimiento hacia el "otro"...

Competencia acerca de sí mismo. Incluye la habilidad de reflexión acerca de la propia cultura, así como la aceptación de la relatividad cultural. (p . 78)

Esta competencia o competencias interculturales son positivas en al menos cuatro esferas: adaptación social, integración cultural, incremento de la idoneidad profesional y salud psicológica referida a las capacidades de integración (Aneas, 2005).

Por otra parte, señala la autora que es difícil que la competencia intercultural se desarrolle, si el ambiente organizacional no promueve la interculturalidad y si estas no están regidas también por un conjunto de principios interculturales. 
doi: http://dx.doi.org/10.15359/ree.18-2.4

URL: http://www.una.ac.cr/educare

CORREO: educare@una.cr

\section{Competencias interculturales docentes y pedagogía intercultural}

La pedagogía intercultural es una respuesta para el ejercicio de la educación en ambientes multiculturales, pues reconoce la diversidad cultural y se centra a partir de ella: "... reconoce que todos somos pluriculturales y nos exige tomar conciencia de nuestros propios valores y creencias ..." (Aguado, 2003, p. 13) en cuanto somos intrínsecamente diversos, múltiples.

Es así como se puede entender la pedagogía intercultural como:

Una alternativa a las propuestas pedagógicas que analizan los fenómenos educativos desde visiones mono culturales, en los que la cultura es un a, monolítica, ya elaborada y delimitada, y la educación es transmisión y perpetuación de dicha cultura única. (Aguado, 2003, p.13)

Cultura única que se impone en la multiculturalidad existente desde la implementación de un currículo que se reconoce así mismo como nacional y básico.

Esto tiene grandes implicaciones, tanto para la pedagogía como disciplina reflexiva de los asuntos educativos, como para las didácticas especificas, aunque hay que reconocer, como lo sugiere Aguado (2003), que todavía falta mucho camino por recorrer en el terreno de la pedagogía intercultural, ya que no hay formulada aún una teoría de la interculturalidad en educación y muchas formulaciones son todavía ideológicas, no hay una metodología educativa específica para este enfoque y sus conceptos estructurantes no están claramente estructurados (Aguado, 2003).

Por estas razones, probablemente, la discusión en la actualidad se ha orientado hacia una reflexión en torno al campo de las competencias interculturales que deben ser instaladas en docentes en sus procesos de formación inicial, como se señalaba al principio de este acápite.

Es así como por competencia intercultural se puede entender:

...las habilidades cognitivas, afectivas y prácticas necesarias para desenvolverse eficazmente en un medio intercultural. Están orientadas a crear un clima educativo donde las personas se sientan aceptadas y apoyadas por sus propias habilidades y aportaciones, así como a permitir la interacción efectiva y justa entre todos los miembros del grupo. (Aguado, 2003, p. 141)

Esta definición operativa de la competencia intercultural $(\mathrm{Cl})$ nos ofrece la integralidad que se busca como concepto estructurante de una investigación sobre competencias interculturales: identifica lo que se debe elaborar, la finalidad, función educativa y el ámbito social en donde debe operar como reguladora de las relaciones sociales al interior del centro educativo y en las aulas.

Entonces, ¿qué debe "tener" un docente en su expertise para concretar el enfoque de competencias interculturales?, y ¿en qué dimensiones debe impactar la formación docente para producir docentes interculturales? 
Ello debe ocurrir en el campo de la práctica docente, en donde estos se apropien de competencias interculturales, entendidas en particular, según Aguado (2003), como los conocimientos, las habilidades o destrezas y las actitudes para operar y favorecer ambientes y relaciones interculturales.

Los docentes interculturales son capaces de conocer a sus estudiantes socioculturalmente, poseen destrezas y habilidades para la integración de estos en las actividades didácticas y educativas y, además, son portadores de actitudes y creencias en las que no se concibe la cultura propia como universal y absoluta. Mediante el modelo Inventario de desarrollo intercultural, Aguado (2003) propone un instrumento que sirve para “... medir de forma objetiva el grado de adquisición de la misma" (p. 142), es decir, de la competencia intercultural.

\section{Contexto de la educación intercultural nacional}

En Costa Rica, los años 70 marcaron el inicio de la discusión en torno a la multiculturalidad, con la creación de centros educativos "bilingüe intercultural" (BI) para la zona indígena de Talamanca. El objetivo de estos centros fue consolidar una educación pertinente a la cultura talamanqueña y reafirmar la identidad étnica. En la actualidad, se cuenta con 34 centros escolares de enfoque Bl. Cabe destacar que para 1992, se crea la ley de Autonomía de los Pueblos Indígenas en la que se discute el desarrollo autónomo, los derechos consagrados en el Convenio OIT N. 169 sobre pueblos indígenas y tribales en países independientes y la creación de la confederación indígena que fundamentan parte de la política educativa intercultural vigente (Moya, 1997).

Para 1994 se crea, en el Ministerio de Educación Pública (MEP), el Departamento de Educación Indígena; posteriormente, entre el 2010 y 2011 cambia su nombre a Departamento de Educación Intercultural, cuyas funciones son la de proponer políticas curriculares, diseñar y evaluar planes y programas de estudio, elaborar recursos didácticos, llevar a cabo procesos de investigación, brindar asesoría técnica, entre otros, en las ofertas educativas dirigidas a los indígenas; además, promover la educación bilingüe y pluricultural en las instituciones educativas de las comunidades indígenas (Poder Legislativo, 2011).

En el 2008, durante el Congreso de Educación Intercultural, se discute el marco normativo internacional, nacional e institucional que regula la materia de educación contextualizada y pertinente, con apoyo de la declaración de la política educativa promulgada por el Consejo Superior de Educación: El centro educativo de calidad como eje de la educación costarricense, cuya visión se inscribe en el desarrollo integral de la ciudadanía, con base en el respeto por la diversidad cultural y étnica; además destaca la responsabilidad de los actuales habitantes con respecto a las necesidades de las futuras generaciones (Consejo Superior de Educación [CSE], 2008).

Debido al avance de la modernidad y a los pasos agigantados de la globalidad, se hace cada vez más necesaria la discusión sobre el tema de la diversidad cultural y, en especial, hablar 
doi: http://dx.doi.org/10.15359/ree.18-2.4

URL: http://www.una.ac.cr/educare

CORREO: educare@una.cr

de una educación intercultural, lo cual no ha sido una labor sencilla, pues implica la modificación de una agenda educativa, un currículo básico y políticas educativas enfocadas a los esfuerzos, al reconocimiento y al respeto de la diversidad cultural, sin perder el objetivo de preparar a los estudiantes para el mundo laboral.

\section{Metodología}

La presente investigación se realiza desde un enfoque cualitativo de alcance descriptivo y centrado en casos, con el fin de obtener una comprensión detallada de las características y experiencias en las prácticas docentes en ámbitos multiculturales. Así mismo, la extracción y producción de esta información se realiza con base en un listado de categorías preestablecidas construidas a partir del análisis bibliográfico de teoría e investigaciones con respecto al tema.

Llevar a cabo esta investigación, desde una perspectiva cualitativa implicó la selección de instituciones de primaria con una condición de multiculturalidad, tanto en zonas rurales como urbanas, públicas, multidocentes, y dirección 2 (259 estudiantes) como mínimo. En total se trabajó en 12 instituciones a nivel nacional con dichas características.

La elección de estas instituciones se hizo tomando como base los datos facilitados por el Departamento de Análisis Estadístico y en coordinación con las Direcciones Regionales de Educación correspondientes de Limón, Coto, Santa Cruz, Nicoya y San José, para producir una lista de centros educativos a visitar, con los que se estableció comunicación para la selección de los informantes.

Para la recolección y producción de la información se recurrió a fuentes primarias, es decir, docentes de aula, alrededor de 97 docentes, entre 6 a 12 estudiantes de orígenes étnicos distintos que cursaran quinto o sexto año, así como directoras y directores (13 en total) de las instituciones, por cuanto son los protagonistas y testigos de experiencias en el abordajes de la gestión curricular que implica realizar acciones para atender la diversidad.

Una entrevista semiestructurada compuesta por 11 categorías y 30 preguntas dirigida al los directores y las directoras, el grupo focal (de 11 categorías y 30 preguntas también) digido al cuerpo docente y un grupo focal (de tres categorías y 20 preguntas) dirigida a estudiantes fueron las principales técnicas para la recolección y producción de datos, pues estas permiten la exploración del discurso en los procesos de educación intercultural.

Obtenida la información, se procedió a realizar una matriz de datos que es la base para la interpretación mediante las categorías de análisis preestablecidas siguientes:

a. Concepto y opiniones sobre el tema: Competencias culturales docentes

b. Acciones realizadas en el aula para atender la diversidad cultural

c. Percepción de la diversidad en el aula 
d. Problemas interculturales que enfrentan en el aula o institución

e. Vacios en la formación recibida

f. Lineamientos, directrices de gestión intercultural que promueven en el aula

g. Propuestas de formación docentes

h. Vacios en la formación recibida

Posteriormente, se abordó la información a través del análisis de contenido basado en el método de comparación constante, ya que permite un contraste entre las afirmaciones y aseveraciones del discurso de los informantes.

\section{Interpretaciones de la diversidad. Competencias interculturales: Habilidades y destrezas}

Se observa que los docentes reconocen y visualizan la existencia de grupos de diversas nacionalidades y culturas dentro del sistema educativo; además muestran la capacidad de describirlos socioculturalmente, lo que permite la aplicación de estrategias que buscan su adaptación/asimilación al proceso educativo. Estas características son mencionadas por Aguado (2003) como algunas de las que pone en práctica diferencialmente un docente intercultural en cuanto busca adaptar o asimilar al estudiante.

Se puede decir que estas características ostentadas por el personal docente son generadas por la vivencia cotidiana en la sociedad actual costarricense, y en la dinámica propia de los procesos de migración hacia el país, por distintas circunstancias. Esto refuerza el concepto de competencia del área, propuesto por Rehaag (2010) en su teoría, en la cual expone que esta se dirige al conocimiento de las culturas de los países de los cuales provienen los estudiantes, competencia que implica su desarrollo en las aulas de las escuelas visitadas.

También se observó que la población docente trata de evitar conductas discriminatorias, lo que favorece que los estudiantes y las estudiantes no se sientan rechazados, sino un poco más de aceptación y apoyo, en búsqueda de una interacción efectiva y equitativa. Se observa, en las visitas, que en las instituciones se da una atención especial al trabajo en valores tales como respeto, igualdad, tolerancia, compañerismo; como marcos de conducta que detienen las actuaciones discriminatorias hacia los mismos estudiantes, sobre todo en relación con las personas de origen nicaragüenses.

Estas actuaciones docentes se relacionan estrechamente con el concepto de competencia intercultural de Aguado (2003), lo que permite decir que existen en forma casi de semilla incipiente en los docentes y las docentes participantes; además, que atañe a la subcompetencia social de Rehaag (2010), la cual remite a la empatía del docente y su capacidad de moderar y resolver situaciones de conflicto. Así se observa, ante estas situaciones, que el cuerpo docente siempre actuó conteniendo y modelando el conflicto, tratando de instruir incluso a través de él. 
doi: http://dx.doi.org/10.15359/ree.18-2.4

URL: http://www.una.ac.cr/educare

CORREO: educare@una.cr

Un elemento muy importante, ligado a lo expuesto anteriormente, es la capacidad de una parte del personal docente de hablar otros idiomas, lo que permitiría la interacción efectiva con las estudiantes y los estudiantes a quienes que se les dificulta el uso del español, o de alguna lengua indígena; lo que se refiere a una de las subcompetencias propuestas por Rehaag (2010) en su teoría de la competencia lingüística, destreza insuficientemente concretada en el cuerpo docente.

Se demuestra que, a pesar del intento de un proceso de integración de las personas culturalmente diversas dentro del aula, existen ciertas percepciones de resistencia hacia esas culturas, como por ejemplo la idea de que los niños y las niñas nicaragüenses vienen con retrasos, o de hogares disfuncionales; ante esto lo que se hace es homogeneizar y equiparar la población al estándar costarricense.

Todo lo anterior permite ver que se cumple, en parte, con una de las dos dimensiones de las competencias interculturales propuestas por Aneas (2005); relacionada con las competencias psicoafectivas del personal docente:este muestra sensibilidad hacia las poblaciones multiculturales presentes en el aula; y las actitudes y las acciones que hace para su atención se basan en un principio de igualdad y solidaridad, el cual es reflejo de la actitud de la población costarricense. Esta situación denota el inicio de una pedagogía intercultural en ciernes, en estado todavía crudo.

A pesar de lo anterior, se reconoce también un gran vacío en cuanto a los procesos de cualificación profesional, la cual se constituye como la segunda dimensión de las competencias interculturales propuestas por Aneas (2005), ya que, a pesar de que las universidades forman al personal docente tomando en cuenta las diferencias de la población que atenderán, no da las herramientas que permitan la especificidad y las especialización en cuanto al tema intercultural. Además de lo anterior, se reconoce que hay una falta importante de capacitación por parte del Ministerio de Educación en cuanto al tema intercultural.

Esta situación es reflejo de la falta de una política educativa referente a lo intercultural; actualmente se tiene docentes hábiles, eficientes y aptos para el desarrollo de sus funciones en contextos multiculturales, aunque no para establecer procesos educativos interculturales, es decir, dialógicos (Aneas, 2005).

Esto ocurre más por una actitud propia humanista equiparadora, que trata de evitar el conflicto, que por directrices emanadas de los jerarcas ministeriales o de lineamientos estratégicos dirigidos a las casa de formación superior, o como resultado de una reflexión profunda en torno a lo intercultural. En este sentido, la misma autora menciona que las competencias interculturales se dan de manera dificultosa, si el ambiente organizacional no las promueve. A través de las participaciones se puede ver que existe cierta dificultad en este aspecto, y se activa la actitud de tratar a todos por igual, sin hacer diferencias, decía una maestra.

De lo expuesto con anterioridad se puede extraer que el personal docente consultado cuenta con algunos elementos mencionados por Aguado (2003) en su concepto de competencia intercultural. Se desprende, entonces, que las habilidades cognitivas, afectivas y prácticas están presentes en las maestras y los maestros; quienes están orientados a crear un ambiente 
doi: http://dx.doi.org/10.15359/ree.18-2.4

URL: http://www.una.ac.cr/educare

CORREO: educare@una.cr

de aceptación, pero desde un punto de vista empírico, regido por la experiencia propia de la persona que imparte la lección y su quehacer diario; más que desde un proceso intelectual guiado por las instancias encargadas.

Esto concuerda con lo expuesto por el mismo autor a través de su modelo de inventario de desarrollo intercultural, quien reflexiona que no existe la plena conciencia intercultural de manera absoluta, pero que tampoco existe la ausencia total de esta (Aguado, 2003).

En este sentido, se podría ubicar la experiencia recogida a través de la realización de este estudio, en una transición entre las etapas de minimización y de aceptación de dicho inventario, ya que discurre entre la visión por parte de algunos docentes de las otras culturas como variaciones de la propia y la aceptación de las otras culturas, aunque estas se perciben complejas.

\section{Aportes a la formación docente}

Si bien, la disposición a trabajar y enfrentar la diversidad cultural tiene una orientación humanista, también ha sido desarrollada de forma espontánea y empírica, lo que, según Rehaag (2010), se esperaría de los cambios en la sociedad actual: un primer paso hacia un nuevo papel profesional del docente el cual trasciende lo mecánico en el aula; que tiene sus bases en la igualdad y enfrenta estas situaciones complejas, cambiantes, inciertas y conflictivas.

Sin embargo, este carácter empírico y humanista del abordaje actual de los docentes demanda que se replantee y modifique metodológica y epistemológicamente el contenido curricular de los programas de estudio de las universidades, así como también las actitudes que poseen los docentes en formación, con el fin de brindar un mejor servicio educativo de acuerdo con las necesidades de esta comunidad diversa, es decir, una educación intercultural.

Según Merino y Muñoz (1998), las acciones realizadas desde la perspectiva docente han sido de dos tipos: uno de tipo funcional, en el cual, se aplica un plan remedial. En la mayoría de las escuelas participantes se observa que estas acciones se realizan con apoyos de otros docentes que experimentaron la misma situación, de los estudiantes que comparten el idioma o cultura, acciones de sensibilización de los estudiantes mediante el conocimiento de los otros culturalmente distintos, actividades extracurriculares que fomentan la convivencia en la diversidad, entre otros. No obstante, con respecto a la respuesta teórica-practica que surge de la investigación de la sociedad actual -segunda vertiente propuesta por estos autores-, no hay evidencia concreta en las escuelas estudiadas.

En el caso de estos docentes, la dificultad para llevar a cabo la complementariedad de estas dos vertientes se debe a que se enfrentan con limitaciones que van desde el tiempo laboral, recargo de funciones, dificultad para dialogar con los padres y madres de familia, escasos o nulos recursos didácticos, metodológicos, y hasta capacitaciones tanto en la universidad, los colegios profesionales e incluso los sindicatos como en su quehacer actual, lo cual se suma a escasos protocolos, lineamientos o directrices para atender estas poblaciones, entre otros. 
doi: http://dx.doi.org/10.15359/ree.18-2.4

URL: http://www.una.ac.cr/educare

CORREO: educare@una.cr

Según Merino y Muñoz (1998), existen dos problemas frecuentes para acoger estos estudiantes de origen distinto: uno de ellos es la adscripción en el centro educativo y el otro la adscripción al aula o al nivel. Esto, por cuanto no se tienen desarrolladas las competencias interculturales, o bien porque el centro educativo o el currículo nacional básico no es flexible en ese sentido.

Se observa en las escuelas visitadas el sobreesfuerzo del docente por realizar ambas acciones, sin que ello signifique un irrespeto de la cultura de origen de estos niños, entonces, ¿cómo llevar a cabo una educación intercultural en un contexto educativo con tales limitaciones y carencias desde la formación inicial docente hasta su trabajo actual?

Desde la perspectiva docente, las acciones que hasta la fecha se han realizado han generado consecuencias positivas tales como mayor empatía y conocimiento por parte de los estudiantes y docentes y, en algunos casos, mayor colaboración por parte de la comunidad educativa. Sin embargo, estas acciones desde el punto de vista conceptual y práctico no son suficientes para atender la diversidad en el aula y centro educativo, se requiere atender y resolver, dicen el cuerpo docente, una serie de necesidades que podrían dar respuesta a la pregunta planteada anteriormente planteada.

Estas necesidades o sugerencias tienen dos orientaciones: desde la formación inicial docente y desde el quehacer actual. A continuación se analizan ambas.

Desde la formación inicial, así como desde el quehacer actual docente se requiere, según Rehaag (2010), de una competencia social, lo que concuerda con lo expresado por los docentes, con respecto a tener mayor conocimiento de aspectos propios de los países de origen, con el fin de comprender el contexto sociocultural del estudiante.

En esta misma línea, la realidad ha hecho evidente la necesidad de hablar un segundo idioma, ya sea inglés o una lengua indígena; por otro lado, en el caso de los estudiantes de habla hispana tales como colombianos, nicaragüenses, salvadoreños, entre otros, es necesario conocer sobre los regionalismos de estas culturas, tal vez por medio de los proyectos de investigación que ahora debe realizar el estudiantado en el marco de alguno de los programas de estudios, por ejemplo en educación cívica, que conforman la comunidad educativa, lo anterior coincide con las competencias lingüísticas que debe poseer el docente, propuestas por Rehaag (2010).

El siguiente aspecto se refiere a la capacidad del docente para brindar al estudiante un sistema educativo de acuerdo con sus necesidades y su cultura. Según los docentes, la universidad es una instancia de una formación teórica descontextualizada de la realidad, lo que prima es la formación en el proceso de enseñanza-aprendizaje estandarizada; entonces, es necesario, según los docentes entrevistados, que se incorpore al currículo universitario ${ }^{6}$ una materia sobre interculturalidad, cuyos contenidos no solo se limiten a lo teórico, sino también

6 El cuerpo docente entrevistado aunque de diferentes edades, refirieron la ausencia de la temática de la interculturalidad en su proceso de formación. Por lo tanto sus opiniones son validas para su experiencia. 
a lo práctico y les brinden, así, herramientas y conocimientos necesarios para el abordaje de la diversidad, por tanto, tal como lo menciona Rehaag (2010), la diversidad debe ser asumida como un proyecto curricular.

Por otro lado, la competencia que se refiere al propio docente, es decir, sobre sí mismo, propuesta por Rehaag (2010), también necesita ser abordada desde la formación inicial docente. Es importante, según los entrevistados, la revisión del sí mismo, de las actitudes, formas de pensar, prejuicios, y estereotipos que se poseen y que generan resistencias y rechazo, los cuales constituyen un obstáculo para trabajar en estas condiciones.

Sereconocetambiénquedesdeelquehacereducativoactual sehafomentadola convivencia e interrelación entre las diferentes culturas a través de actividades extracurriculares, festivales y demás, donde se han promovido valores como comunicación, cooperación, igualdad, respeto, aceptación mutua, indispensables, según Rehaag (2010), para inspirar principios pedagógicos y para llevar a cabo una educación intercultural; sin embargo, cabe aclarar que algunos centros educativos manifestaron la dificultad de no tener una guía o lineamientos claros o bien la poca flexibilidad del centro educativo para la realización de estas actividades.

En lo referente a las redes de apoyo para atender adecuadamente a la población inmigrante, se han realizado, en algunas escuelas visitadas, reuniones con diversas instancias como Dirección Regional de Educación correspondiente; establecimiento de apoyo por parte de profesionales en problemas emocionales, de conducta y aprendizaje; otros docentes de la misma institución. No obstante la ayuda por parte del MEP al tema de la diversidad, esta ha sido escasa, esto por cuanto no hay un discurso intercultural claro.

En síntesis, ante el cambio actual del contexto educativo costarricense, se genera una llamada de atención orientada a una reforma en el perfil docente desde sus cimientos. En la universidad, principalmente, es importante el desarrollo de competencias interculturales en todas sus dimensiones, desde el sí mismo y conocimiento del otro, así como herramientas y conocimientos teóricos y prácticos que garanticen una prestación de servicios educativos interculturales y, por ende, se desarrolle un clima educativo de reconocimiento y aceptación de la diferencia (Aguado, 2003).

\section{Una pedagogía intercultural o la exaltación de la diferencia}

La pedagogía intercultural como modelo educativo es relativamente reciente, lo más cercano que se observa son las estrategias educativas relacionadas con la otredad desde la compensación, que es lo que se pudo identificar en las experiencias españolas revisadas.

A escala nacional, y desde el año 2000, en el país se ha venido investigando en torno a las formas de enfrentar la diversidad cultural en el aula. El estudio en torno a la escasa preocupación del docente respecto de la diversidad en el trabajo de Campos, Castro y Ceciliano (2005); la práctica educativa homogenizadora o sin un referente conceptual intercultural, como lo indican 
doi: http://dx.doi.org/10.15359/ree.18-2.4

URL: http://www.una.ac.cr/educare

CORREO: educare@una.cr

Artavia y Cascante (2007), dado el currículo basado en contenidos en el que fundamenta su accionar educativo; o por la observación de Coto, Ledezma y Vargas (2009) de que lo niños migrantes buscan reprimir su identidad (modelo asimilacionista) se intenta establecer, entonces, una estrategia metodológica centrada en el diálogo -más conceptualizado como resolución de conflictos que intercultural- que permita atenuar conflictos, como la forma de atender el tema de la diversidad cultural en el aula. Su análisis implica cambios curriculares relevantes.

En esta dirección, Vargas (2011) planteó la actuación desde el campo de lo curricular y lo pedagógico y en relación con los conceptos interculturales. Por otra parte, Araya y Hernández (2011) atendieron el fenómeno intercultural desde la sobrevaloración de lo cognitivo de los estudiantes, en cuanto a que el rezago aparece como un dato de que el estudiante requiere un tratamiento especial, entonces, la diferencia cultural se reduce a un problema de rezago educativo. Y por esta vía se integra al otro, al migrante, reduciéndolo a alguna categoría de atención diferenciada.

En cuanto los procesos unidimensionales o bidimensionales, planteados en el apartado conceptual, en relación con las prácticas educativas, estas se articulan desde el aporte que la cultura nacional le da al migrante, pero no toma en cuenta el aporte que este puede dar (modelo bidimensional).

El aporte se implementa desde la gestión docente con su avasalladora fuerza homogeneizadora, proceso observado en el trabajo de campo en estas escuelas. Por otro lado, el otro se ve compelido a integrarse/asimilarse a la cultura receptora, proceso que se da por medio de la represión de la cultura propia y aceptación de la cultura receptora, según la matriz de Berry, citada por Núñez (2009), en la que se articulan procesos de asimilación con los del "Melting Pot" y se concreta un modelo de aculturación basado en el currículo -del cual no se puede salir-, la diferencia queda subsumida, entonces, en la norma de la escuela a la que los extranjeros se tienen que someter, decían en una de las escuela visitadas.

El currículo es el marco desde el cual el cuerpo docente modela su práctica educativa sin saber que su actuación materializa un modelo de aculturación: educar para igualar, que persigue occidentalizar (García, Pulido y Montes, 1997) o costarriqueñizar, modelo que realiza esta operación mediante la compensación y homogenización de la multiculturalidad.

El problema surge cuando, ante la realidad multicultural, no se es capaz de valorar en cuanto tal y se procede, por un lado, mediante una gestión educativa igualitaria, pero con un efecto asimilacionista, o bien, se genera un práctica educativa compensatoria (Aguado, 2003), es decir, la diferencia cultural se trata como una deficiencia.

Las dos estrategias juntas se convierten, entonces, en una negación absoluta de cualquier posibilidad para el desarrollo de una pedagogía intercultural; puesto que, a diferencia de esta, que valora la diferencia y la exalta para enriquecer la práctica educativa, y por esta vía el estudiante se puede volver un actor protagónico del acto educativa; se sacraliza la otra vía, en la que el protagonista es el docente, quien desde el orden curricular opera la estandarización, 
territorio simbólico de la práctica docente, que se apega a los programas, a las minutas, a los planeamientos y a las normas de la escuela.

Ello por cuanto el cuerpo docente se enfrenta a esta realidad desde una visión monocultural, desde la visión propia del currículo estándar establecido por planes y programas de estudio iguales para todos, currículo que homogeniza tantolos conocimientos que se transmitirán como los valores sociales y culturales que el estudiantado debe incorporar en su bagaje. La educación está hecha, a pesar del multiculturalismo presente, para producir ciudadanos costarricenses.

Una actitud tolerante de base permite una gestión educativa igualitaria, que intenta no hacer diferencias entre los estudiantes y tratarlos a todos igual. Esta actitud básica de respeto a los otros culturales que se funda en una consideración ética basada en la presunción de que todos somos iguales, se concreta en una pedagogía que no discrimina, al menos no de modo evidente; sino todo lo contrario, intenta integrarlo a un todo establecido de antemano, en donde a todos los mezclamos y sale del caldero una identidad homogénea. No obstante, la educación intercultural presupone también el reconocimiento y valorización de la diferencia, por lo que un trato igualitario, aunque afectuoso, puede borrar las diferencias.

Tal gestión no identifica la operación de estandarización cultural, porque con base en la idea de que se respeta al otro, finalmente la práctica curricular en el aula logra hacer que todos se sientan iguales; no obstante, en muchas de estas instituciones se realizan actividades extracurriculares con la finalidad de generar espacios de comprensión de la diversidad, pero que facilitan la integración del estudiante como proceso educativo igualitario.

La multiculturalidad es un hecho dado, irrefutable; tanto las estadísticas nacionales respecto de la presencia en las aulas de extranjeros, grupos culturales y etnias criollas, como en los antecedentes revisados y en las escuelas visitadas, se ha observado la visión que de esta tienen directores, docentes y estudiantes. Visión que de una manera general se puede calificar como positiva. Y se podría señalar, porque se vio en el trabajo de campo, que existe una pedagogía intercultural que se inicia desde la práctica misma e interacción docente con la diversidad, pero esta tiene como misión disolver la diferencia.

Una pedagogía intercultural debería, al menos, identificar y operacionalizar desde la realidad multicultural la diferencia, exaltarla y transformarla en actividades de aula que favorecen no solo la diversidad, sino que enriquecen el acto educativo como tal. Lo que se observó en las instituciones educativas visitadas es una operación en la que la multiculturalidad real se diluye en una actitud que exalta la igualdad; todo lo contrario de las propuestas interculturales en las que incluso en espacios de aula, en que no existiera la multiculturalidad, a esta se la debe exaltar, porque todos somos distintos (Aguado, 2003). 
doi: http://dx.doi.org/10.15359/ree.18-2.4

URL: http://www.una.ac.cr/educare

CORREO: educare@una.cr

La pedagogía intercultural como práctica dialógica se desarrolla a partir del favorecimiento que se tiene que realizar desde la gestión del currículo y desde la práctica docente de la identidad cultural (dimensión cultural), de la valoración y respeto (dimensión axiológica) y desde los programas de estudios (dimensión praxiológica) (Merino y Muñoz, 1998); es decir, reconocer que la diversidad cultural es su centro (Aguado, 2003). Ahora bien, ya señalada anteriormente, esta pedagogía intercultural se concreta y se operacionaliza por medio de competencias especificas que no se encuentran hoy día en la práctica docente y son una carencia observable.

La práctica educativa debe sufrir y experimentar cambios positivos en una dirección que supere las carencias técnicas específicas observadas. Un cambio de enfoque es necesario para orientar la acción educativa hacia una serie de consideraciones importantes de cara a entender:

- La diferencia cultura como foco de reflexión

- La diferencia como norma

- La diferencia como superávit cultural y no como déficit por compensar/adecuar

- Este conjunto de cambios y de miradas alternativas se deberían condensar en habilidades y destrezas pedagógicas capaces de exaltar la diferencia. Todo lo contrario a lo que sucede hoy en las aulas nacionales, no en cuanto la multiculturalidad como hecho dado, sino en cuanto al tratamiento curricular.

No obstante, hay algún camino recorrido en cuanto a la pedagogía intercultural, no se parte de cero, sino de un punto intermedio, pedagogía que se verifica en el diario vivir de los docentes y las docentes; sin embargo, se carece de una reflexión de fondo al respecto. Tal avance se ve en la calidad afectiva de docentes hacia sus estudiantes, en los avances alcanzados por la educación indígena y en el marco legal progresivo. Falta encontrarse dialógicamente con la diversidad, tal tarea quedará bajo la responsabilidad del campo de la pedagogía intercultural.

\section{Conclusiones}

La mayoría del personal docente cuenta en su práctica educativa con la dimensión psicoafectiva de las competencias interculturales. Es decir, todos los niños y las niñas son tratados con afecto, desde una mirada igualitaria que no busca ejercer diferencias en el aula.

Las competencias que más se desarrollan son aquellas encaminadas a la competencia social, ya que la población docente participante se preocupa por generar empatía y entendimiento entre estudiantes cultural o nacionalmente distintos y hacia estos, y moderar 
algunas situaciones de conflicto intercultural. No hay, sin embargo, en la práctica docente una reflexión de orden intercultural ni se manejo el enfoque pedagógico intercultural, este no está presente en la práctica docente.

Se denota también que la falta de lineamientos de las autoridades políticas ministeriales y nacionales respecto de una educación intercultural produce agujeros en la formación en interculturalidad en los procesos de formación inicial y continua del docente, así como en la aplicación de procesos de capacitación en servicio que podrían solventar dichos lineamientos.

El desconocimiento en la atención de la diversidad cultural se arrastra desde la formación inicial ${ }^{7}$. Las universidades públicas no brindan a nivel de bachillerato, en I y II ciclo, formación en pedagogía intercultural. Y solo en la Universidad Nacional y en la Universidad Estatal a Distancia se observa un curso de esta temática en el nivel de licenciatura. Por otro lado, no tenemos evidencia en este momento de que se haya adoptado el enfoque intercultural en los procesos de formación docente en las carreras de educación de I y II Ciclo. Es por ello que se hace necesario la revisión y modificación de las mallas curriculares de las carreras de educación, como respuesta a la realidad culturalmente distinta que se percibe actualmente en el ámbito nacional.

La dificultad principal expresada por los docentes no radica en la percepción de la diversidad desde el punto de vista humano o social, sino desde una gestión educativa que no genera diferencias curriculares y cuyo resultado es la asimilación o integración del otro a la cultura. Esto evidencia que existe poco o nulo reconocimiento de la diversidad en el proceso enseñanza-aprendizaje, de manera que el modelo o enfoque que poseen los docentes es resultado de una formación inicial docente orientada a la estandarización de la población sin tomar en cuenta las características culturales particulares, diferentes de las establecidas en la ley 7600 , con respecto a las personas en condición de discapacidad.

En resumen, la práctica docente en la actualidad carece de la capacidad de actuación ante la diversidad cultural existente en las aulas desde un enfoque que la favorezca, que la utilice como un medio educativo. La diversidad cultural no es un valor consciente en el diario acto educativo de aquellas instituciones en las que la multiculturalidad es un hecho evidente.

Trascender de instituciones en donde la multiculturalidad es real y un punto de partida positivo hacia entornos educativos interculturales no es una opción hoy sino una obligación ética, y se están presentando excelentes oportunidades para ello de estimulo de una educación intercultural tanto en los espacios y opinión pública como en el ambiente del Ministerio de Educación, que ha creado para esos propósitos un departamento especializado, además de la implementación de una serie de acciones al respecto.

7 Una lectura rápida de los planes de estudios de las universidades públicas revela la ausencia de formación en pedagogía intercultural en el nivel de bachillerato. 
doi: http://dx.doi.org/10.15359/ree.18-2.4

URL: http://www.una.ac.cr/educare

CORREO: educare@una.cr

\section{Recomendaciones}

$\checkmark$ Conocer la realidad de las poblaciones migrantes para poder entender sus conductas. Es una necesidad latente mejorar la enseñanza en interculturalidad y mejorar la capacidad didáctica de atención de esta (talleres con información de otros países culturas) como medio de sensibilización y profesionalización intercultural.

$\checkmark$ El estudiantado de diversas culturas que comparte y convive diariamente forman naturalmente redes de pares. Fortalecer estas redes de apoyo entre estudiantes, mediante la organización intencional de estas, para generar una cultura educativa amplia, puede ser un factor muy enriquecedor de valoración de la diversidad.

$\checkmark$ Incorporar en el currículo espacios educativos para conocer otros espacios sociales y culturales, e integrar a los niñas y las niñas y a sus padres y madres en estos para que haya encuentros sobre la cultura y las culturas presentes en un momento determinado en una institución educativa, puede ser un medio muy eficaz de reconocer y dialogar con la diversidad. No obstante, no se trata que sea solo en el Día de las Culturas o en un acto cívico institucional en donde se celebre la interculturalidad, sino de propiciar actividades curriculares más permanentes.

$\checkmark$ Trabajar durante el año en la organización de varios días culturales sería muy útil, entendidos estos días desde un marco curricular más amplio, como el de un proyecto de centro. Las semanas de convivios interculturales son en este marco un potente medio de reconocimiento, y las visita a las escuelas de los padres y madres de estudiantes de otras culturas, y que estos traigan sus comidas, hablen de su país de origen, y compartan sus valores son un factor de autoestima muy fuerte para los niños y las niñas de otras culturas, como se evidencia en las escuelas de Cahuita y Puerto Viejo de la región Educativa de Limón. Estas experiencias son un punto de partida interesante para que se desarrollen en otras escuelas nacionales.

$\checkmark$ Por otro lado, es necesario, brindar al personal docente los instrumentos pedagógicos, didácticos y tecnológicos necesarios (dinámicas participativas, convivios interculturales, invitación a padres y madres de familia para hablar de su cultura, proyectos de investigación, círculos de estudios, etc.) para trabajar la diversidad e interculturalidad en el aula y centro educativo. Se le deben mostrar y capacitar en los instrumentos y materiales didácticos que puede usar. También, observar de otras instituciones las experiencias vividas al respecto.

$\checkmark$ Los estudiantes han abogado por clases más participativas, al aire libre, más interacción social, y aprovechar esos espacios curriculares para trabajar académicamente la diversidad cultural.

$\checkmark$ Más instrucción en pedagogía y didáctica intercultural, talleres, cursos de capacitación en servicio, uso de video relativos al tema, conocimiento de las experiencias de otros países, porque entre más se nos instruya, más se nos va alivianar el trabajo para la atención de la interculturalidad. 
doi: http://dx.doi.org/10.15359/ree.18-2.4

URL: http://www.una.ac.cr/educare

CORREO: educare@una.cr

Por otro lado, resulta evidente que el personal docente debe hacer una revisión profunda de las actitudes que tiene con respecto a la población estudiantil que presenta diversidad cultural; una revisión intencionada a validar la diversidad cultural, al desarrollo de una actitud más abierta para con sus estudiantes cultural y nacionalmente distintos.

$\checkmark$ La gestión administrativa, mediante los aportes de la Dirección Curricular, el Departamento de Educacion Intercultural, el de Estudios e Investigación Educativa, de Educación Indígena, además de directrices de los despachos del Ministro y Viceministros que fortalezcan, como ha sido la tónica, la educación intercultural y el aporte del cuerpo de directores institucionales son sumamente importantes para transformar interculturalmente la educación. Estos son los que deben generar directrices claras dirigidas a la comunidad educativa para que tengan como fin regular los procesos relacionados con el tema intercultural.

$\checkmark$ La vida institucional se enmarca en la institucionalidad del Ministerio de Educación Pública. A este le toca generar políticas educativas concernientes al tema de la educación intercultural, que se conviertan en un punto de referencia a nivel nacional y que permitan un abordaje integral de los procesos que se dan en los contextos multiculturales, de manera que las actividades que se ofrecen en los centros educativos no se aborden desde una base empírica, sino desde una intencionalidad concreta. Por otra parte, tiene una amplia responsabilidad en los procesos de formación continua o en servicio junto al rol de las universidades públicas y privadas en cuanto a formación inicial y las acciones de evaluación y seguimiento.

$\checkmark$ En cuanto a estas, urge que las carreras de educación integren cursos y procesos de formación en pedagogía intercultural que preparen al cuerpo docente para su salida al campo laboral con estas competencias. Por otro lado, es urgente también la coordinación de sesiones de trabajo de las universidades con el Ministerio de Educación para determinar y definir los perfiles de docentes por contratarse por el Ministerio en cuanto a las competencias interculturales que deben portar de cara a los cambios que se han venido realizando en los planes y programas de estudio nacionales.

$\checkmark$ Que las universidades incorporen a su programa de estudios mayor trabajo de campo, talleres, convivios y giras hacia las distintas regiones del país que presentan una amplia gama cultural con el fin de sensibilizar a los futuros docentes de esta realidad que eventualmente tendrán que enfrentar.

$\checkmark$ Este conjunto de recomendaciones no son exhaustivas, solo indicativas de lo que parece que urge hacer en el terreno de la pedagogía intercultural, tanto por parte docentes, direcciones institucionales, estudiantes, universidades y Ministerio de Educación Pública, de cara al futuro, el que lejos de homogeneizarse tiende a la diferenciación social y cultural cada vez mayor. 
doi: http://dx.doi.org/10.15359/ree.18-2.4

URL: http://www.una.ac.cr/educare

CORREO: educare@una.cr

\section{Referencias}

Aguado, T. (2003). Pedagogía intercultural. Madrid: McGraw-Hill.

Aneas, M. A. (2005). Competencia intercultural, concepto, efectos e implicaciones en el ejercicio de la ciudadanía. Revista Iberoamericana de Educación, 35(5), 1-9. Recuperado de http:// www.rieoei.org/delosleclncluyetores/920Aneas.PDF

Araya, G. y Hernández, S. (2011). La interculturalidad en escuelas costarricenses con población migrante. Revista Actualidades Investigativas en Educación, 11(1)1-28. Recuperado de http:// revista.inie.ucr.ac.cr/uploads/tx magazine/interculturalidad-escuelas-costarricensesaraya.pdf

Artavia, C. y Cascante, L. (2007). Competencias de las y los docentes para la atención de la diversidad intercultural dentro del proceso edcuativo en el Liceo Otilo Ulate Blanco de San isidro de Alajuela (Tesis de maestría inédita). Universidad Nacional, Heredia, Costa Rica.

Campos, L., Castro, G., y Ceciliano, A. (2005). Estudios sobre la opinión de diversidad cultural de los niños y niñas de quinto grado de la escuela Juan Rafael Chacón ubicada en Boca Arenal, Cutris de San Carlos de Alajuela. (Tesis de licenciatura). Universidad Nacional, Heredia, Costa Rica.

Consejo Superior de Educación. (2008). El centro educativo de calidad como eje de la educación costarricense. Recuperado de http://dinamico.uned.ac.cr/ece/images/documents/ doc2011 yrivera/un centro educativo de calidad.pdf

Coto, R., Ledezma, Y. y Vargas, K. (2009). Proyecto de estrategias metodológicas que puedan implementarse en el primer ciclo de la escuela de Santo Tomás de Santo Domingo de Heredia, para un abordaje de la diversidad cultural desde una perspectiva creativa (Tesis de licenciatura inédita). Universidad Nacional, Heredia, Costa Rica.

Escalante, C., Fernández, D. Gaete, M. (2012). Ejercicios docentes en contextos multiculturales: Lecciones para la formación en competencias docentes interculturales. San José, Costa Rica: MEP. Recuperado de http://www.mep.go.cr/sites/default/files/competencias interculturales.pdf

García, F. J., Pulido, R. A. y Montes, Á. (enero-abril, 1997). La educación multicultural y el concepto de cultura. Revista Iberoamericana de Educación, 13. Recuperado de http://www.oei.org. co/oeivirt/rie13a09.pdf

Merino, J., y Muñoz, A. (mayo-agosto, 1998). Ejes de debate y propuestas de acción para una pedagógía intercultural. Revista Iberoamericana de Educación, 17. Recuperado de http:// www.rieoei.org/oeivirt/rie17a07.htm 
Moya, R. (enero-abril, 1997). Interculturalidad y reforma educativa en Guatemala. Revista Iberoamericana de Educación, 13, 129-155 Recuperado de http://www.rieoei.org/oeivirt/ rie13a06.pdf

Nuñez, I. (2009). La adaptación de currículo a la diversidad cultural. Revista lberoamericana de Educación, 49(2), 1-9. Recuperado de http://www.rieoei.org/deloslectores/2861Nunez.pdf

Poder Legislativo (23 de octubre de 2011). Decreto № 36451-MEP. La Gaceta No 48. Recuperado de http://www.gaceta.go.cr/pub/2011/03/09/COMP 0903 2011.html\# Toc287347865

Rehaag, I. (marzo-abril, 2010). La perspectiva intercultural en la educación. El Cotidiano, 160, 75 83. Recuperado de http://www.redalyc.org/articulo.oa?id=32512766009

Vargas, M. (2011). Estrategias didácticas para la favorecer el desarrollo integral de niños y niñas de cuatro años, desde un enfoque de la educación intercultural, Cóbano Puntarenas. (Tesis de maestría), Universidad Nacional, Heredia, Costa Rica.

\section{(6) Cómo citar este artículo en APA:}

Escalante, C., Fernández, D. y Gaete, M. (mayo-agosto, 2014). Práctica docente en contextos multiculturales: Lecciones para la formación en competencias docentes interculturales. Revista Electrónica Educare, 18(2), 71-93.

doi: http://dx.doi.org/10.15359/ree.18-2.4

Nota: Para citar este artículo en otros sistemas puede consultar el hipervínculo "Como citar el artículo" en la barra derecha de nuestro sitio web:

http://www.revistas.una.ac.cr/index.php/EDUCARE/index 